\title{
Element stage processing system for microphone array calibration
}

\author{
Feng Jiang and Xiaodong Li \\ Institute of Acoustics, China Academy of Sciences, Beijing, 100080, P.R. China
}

(Received 29 April 2003, Accepted for publication 12 June 2003 )

Keywords: Microphone array, Channel mismatching, Cluster algorithm, Principle component analysis, Tolerance prediction PACS number: 42.60.G, 43.38.K [DOI: 10.1250/ast.25.112]

\section{Introduction}

Microphone array and its application have become very active topics in the field of speech and audio processing. The problem how to theoretically design microphone array with excellent performance has been discussed in many documents $[1,2]$. In practical terms, the directivity pattern of microphone arrays and their performances will be distorted due to errors in critical components. These errors are caused by variations in the characteristics of the microphone elements, the analog amplifiers, and anti-aliasing filters, another source of errors arising from the non-synchronous sampling.

Therefore, another thing worth attention is how to actually realize the results of theoretical design, in which how to solve the problem of channel mismatching has always been a focus in the research on microphone array application technology. Our research on the above mentioned problem is divided into three stages: 1) element stage processing (ESP) based on standing-wave calibration, 2) post-beamformer processing on tunable system, 3) in situ processing on installation platform.

The achievements of our research on the first stage are reported in this paper, which particularly considers the channel mismatching errors, with regardless of the uncertainty in element position. After introduced realization principle of ESP, effective experimental results will be discussed.

\section{Principle}

How great the influence of channel mismatching on the performance of microphone array is? Whether the requirements of microphone array and vector microphone array on channel mismatching are same? What range of channel mismatching error needs correction? How to automatically select microphones with satisfactory accuracy from a large number of samples?

Inspired by the above mentioned problems, this paper propose the conception of ESP (element stage processing) method. Schematic diagram of ESP and its software flowchart are respectively shown in Figs. 1 and 2. The difference between ESP method and the traditional channel mismatching measuring method is that ESP organically combines computer aid test (CAT), computer aid design (CAD), tolerance predication and channel mismatching correction together, and thereby quite satisfactorily solves the above mentioned problems.

\section{Experiment result}

ESP system has been used to test hundreds of microphone samples, and effective experiment results have been obtained.
Figure 3 shows scatter diagrams of channel mismatching of model-A microphone ( 9 frequency bin), the horizontal ordinate represents group time-delay and the vertical one represents relative amplitude sensitivity, As is shown in Fig. 5, symbol " $\bigcirc$ " denotes sample 1 , symbol " $\square$ " denotes sample 2 , and symbol " $\square$ " denotes sample 3, and so forth. As is show in Fig. 4, the performance of scatter diagrams can be improved by using principle component analysis (PCA) [3].

Figures 3 and 4 illustrate that sample 1, 2, 3, 5, 6, and sample 10 have good consistence, are suitable for building large-scale microphone array. It is confirmed by clustering result in Fig. 5. The numbers along the horizontal axis in Fig. 5 represent the indices of microphone samples. The links between samples are represented as upside down U-shaped lines. For example, the link between sample 2 and 10 represents they have very small uncertainties in amplitude and phase errors.

The effects of channel mismatching errors actually obtained can be evaluated with the aid of the simulated directivity pattern. According to the channel error distribution probability of type B microphones, an error-bar diagram is used to get the tolerance prediction of nine-element microphone array. In Fig. 6, beam performance is worsened and the average side-lobe level is increased to $-14 \mathrm{~dB}$. To keep the directivity pattern acceptable, the necessary corrections are required. Minimizing the range of phase errors will reduce the possible distortions of the directivity pattern.

The correction method of ESP system is efficient and easy to be implemented. Since speaker localization requires quite high phase consistency, ESP system is used to correct channel mismatching for model $\mathrm{C}$ microphone samples. Measurement results before and after correction are respectively indicated in Figs. 7 and 8. The channel phase mismatching after correction is lower than $1.0 \times 10^{-6}$ degree.

\section{Summary}

Through combining the array design, element measurement, tolerance predication and error correction together, this paper has established an automatic integration environment, i.e. ESP system, and achieved satisfactory experiment effects. The following are some other conclusions about the experiment:

1) For the automatic classification of microphones, the channel mismatch scatter diagram proposed in this paper is a simple and straightforward classification tool characterized by man-machine interaction, which is used to design the initial value of clustering in sensor classification and avoid falling 


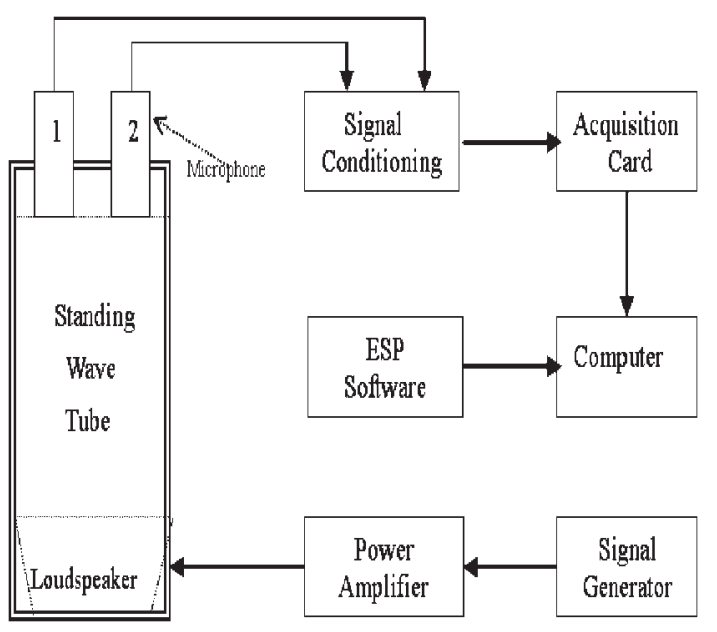

Fig. 1 Block diagram of ESP system.

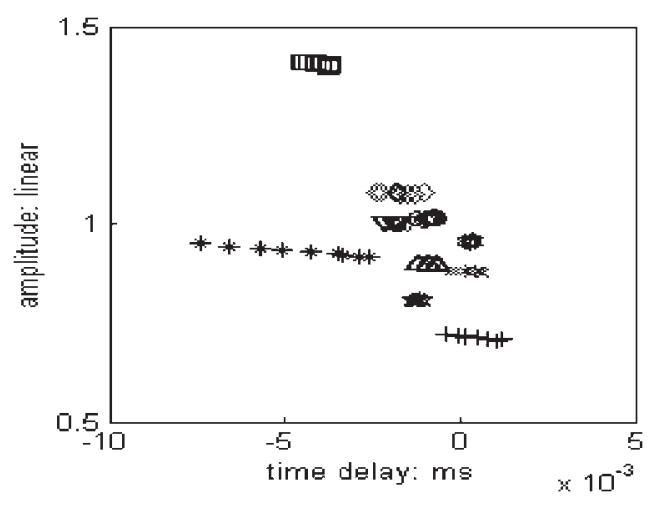

Fig. 3 Scatter diagram of channel mismatching.

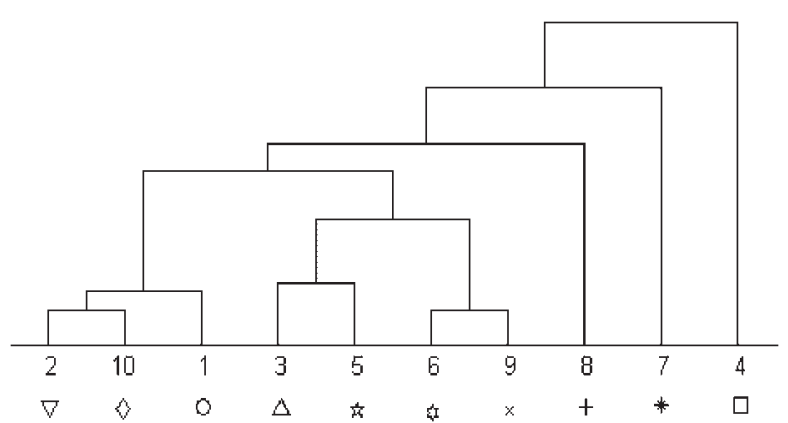

Fig. 5 Hierarchical tree of cluster result.

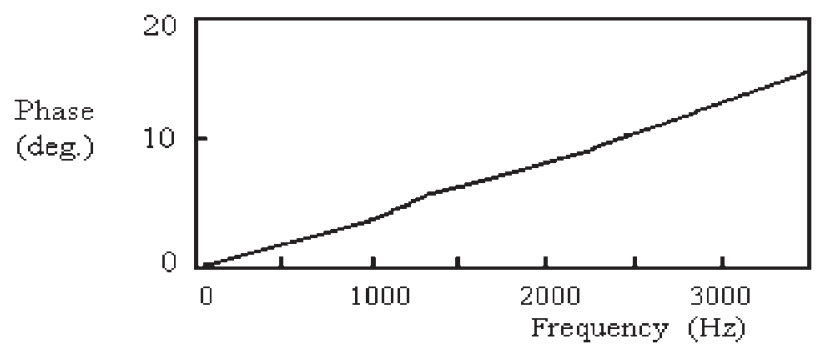

Fig. 7 Phase mismatching before correction.

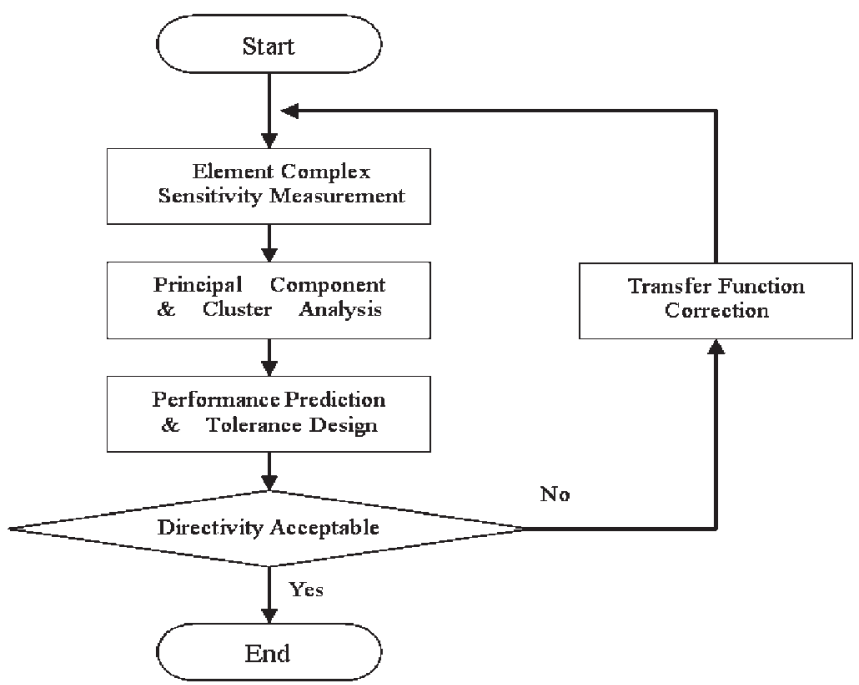

Fig. 2 Flowchart of ESP software.

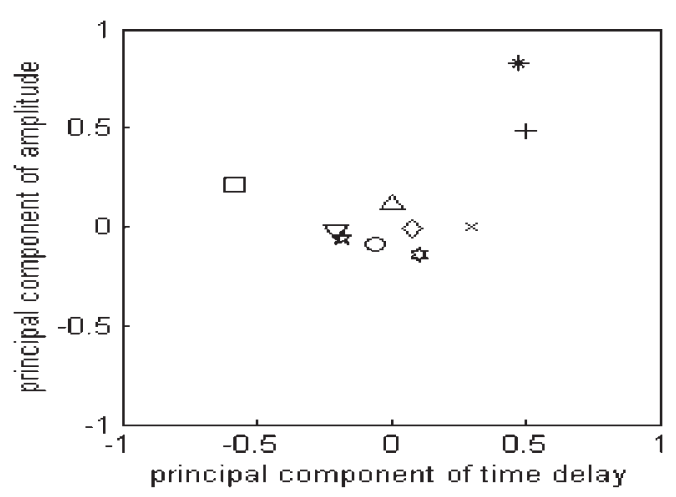

Fig. 4 Principal component analysis.

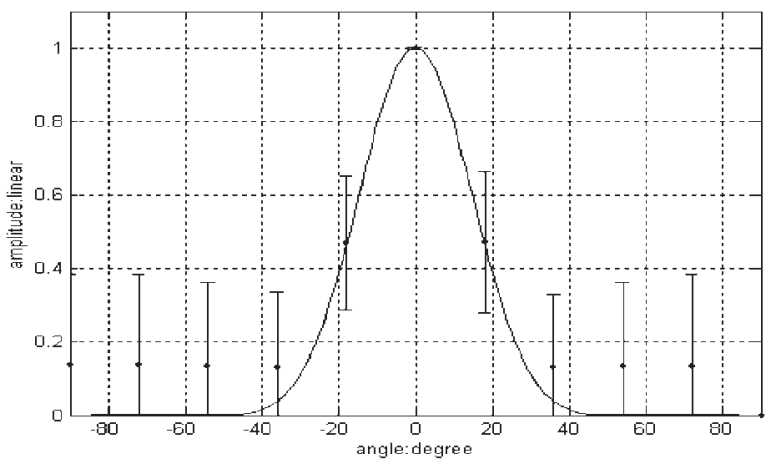

Fig. 6 Error-bar diagram of array beam.

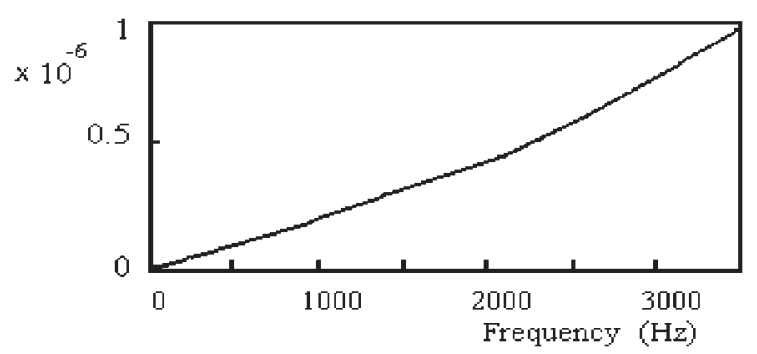

Fig. 8 Phase mismatching after correction. 
into local minimum value in iterative cluster algorithm.

2) The result of using ESP system for microphone array design indicates that Taylor-window function has low sensitivity to channel mismatching. For 15-element microphone array, channel phase mismatching is required to be lower than 0.5 degree to obtain array beam with side-lobe level under $-40 \mathrm{~dB}$ (Tolerance error: $5 \mathrm{~dB}$ ).

\section{References}

[1] R. W. Stadler, "On the potential of fixed arrays for hearing aids," J. Acoust. Soc. Am., 94, 332-342 (1993).

[2] R. T. Williams, "An improved spatial smoothing technique for bearing estimation in multipath environment," IEEE Trans. Acoust. Speech Signal Process., 36, 425-432 (1988).

[3] S. K. Kung, "Adaptive principal component extraction and application," IEEE Trans. Signal Process., 42, 1202-1217 (1994). 